\title{
Sken\&agraphie
}

SKIINagraphII Coulisses des arts du spectacle et des scènes

émergentes

5 | 2018

Juste la fin du monde, de Lagarce à Dolan

\section{Approche génétique du régime de disposition textuelle dans Juste la fin du monde}

\section{Pascal Lécroart}

\section{OpenEdition}

\section{Journals}

Édition électronique

URL : http://journals.openedition.org/skenegraphie/1391

DOI : 10.4000/skenegraphie.1391

ISSN : 2553-1875

\section{Éditeur}

Presses universitaires de Franche-Comté

\section{Édition imprimée}

Date de publication : 1 janvier 2018

Pagination : 61-69

ISBN : 978-2-84867-5609-8

ISSN : $1150-594 \mathrm{X}$

Référence électronique

Pascal Lécroart, « Approche génétique du régime de disposition textuelle dans Juste la fin du monde », Sken\&agraphie [En ligne], 5 | 2018, mis en ligne le 01 janvier 2019, consulté le 30 avril 2019. URL http://journals.openedition.org/skenegraphie/1391 ; DOI : 10.4000/skenegraphie.1391

Ce document a été généré automatiquement le 30 avril 2019

Presses universitaires de Franche-Comté 


\title{
Approche génétique du régime de disposition textuelle dans Juste la fin du monde
}

\author{
Pascal Lécroart
}

1 On tend régulièrement à rassembler l'œuvre dramatique de Jean-Luc Lagarce autour de certains traits caractéristiques d'ensemble: un théâtre de la parole, plus épique que dramatique, centré sur la difficulté du dire qui rend souvent opaque le récit derrière le ressassement. Inversement, en peu d'années, Lagarce a manifesté une capacité à se renouveler et à expérimenter, malgré certaines redites favorisées par l'absence de perspectives éditoriales pour bon nombre des œuvres, encourageant les reprises et les réécritures. On peut néanmoins repérer des axes directeurs pluriels permettant d'organiser et de structurer l'ensemble de l'œuvre.

2 Juste la fin du monde (1990) se situe au croisement de ces différentes perspectives. Typique d'un théâtre du discours presque dépourvu d'action, l'œuvre est généralement située dans le prolongement de Retour à la citadelle (1984) et de Derniers remords avant l'oubli (1987). Elle annonce par ailleurs J'étais dans la maison et j'attendais que la pluie vienne (1994) - qui reprend la thématique du retour du fils dans sa famille mais d'un autre point de vue - et, surtout, Le Pays lointain (1995) qui, étant donné l'absence de toute lecture publique, représentation ou édition de Juste la fin $d u$ monde, en était la réécriture et l'accomplissement. Imaginons que Lagarce ait vécu : qui sait s'il aurait voulu, après Le Pays lointain, redonner éditorialement ou scéniquement sa place à une œuvre désormais presque entièrement absorbée dans une autre?

3 Pourtant, par un trait assez singulier et peu mis en évidence, Juste la fin du monde doit être mis en rapport avec les deux œuvres qui la précèdent dans l'écriture, d'esprit a priori bien différent: Music-hall, écrit en 1989, et Histoire d'amour (Derniers chapitres) écrit vraisemblablement entre la fin de 1989 et le début de 1990. Ce sont les seules œuvres où, formellement, Lagarce fait usage d'une écriture qu'on peut dire en versets, avec de fréquents retours à la ligne non matérialisés par la majuscule ou par l'alinéa. Même si, dans la plupart des cas, le vers tient sur l'espace de la ligne, on évitera de parler de vers 
libres dans la mesure où ils constituent de courts paragraphes quand ils dépassent la ligne. Certes, dès Carthage, encore ou Noce, on trouve des répliques - le plus souvent longues - organisées en paragraphes; mais il s'agit alors d'une structuration avant tout logique - le discours entendu à la radio dans Carthage, encore -, cohérente avec la structure syntaxique. Le terme de paragraphe est alors plus satisfaisant que celui de verset. En revanche, à partir de Music-hall ${ }^{1}$, Lagarce se permet des retours à la ligne qui, dans la longue et tardive postérité du verset claudélien, constituent, par leur fréquence, une rythmique verbale singulière. Certes, à l'inverse de Claudel, Lagarce n'a pas laissé de texte critique où il s'expliquerait sur ce choix dispositionnel du texte ; rien n'indique, par exemple qu'il faille marquer une interruption en fin de verset ou respirer. Il parait néanmoins évident que la disposition engendre une compréhension et une appropriation spécifiques du texte, d'abord lors de la lecture individuelle dans le mouvement même de l'œil, contraint à un mouvement spécifique, ensuite dans la restitution à voix haute par l'acteur, nécessairement influencée et guidée au moment de l'apprentissage du texte.

Ce type d'écriture en versets aura une pérennité plus limitée qu'il ne parait: si on retrouve, de Nous les héros au Pays lointain en passant par J'étais dans la maison..., des retours à la ligne réguliers dans les interventions de personnages, ils n'ont plus la fréquence qu'ils avaient dans les trois pièces de 1989-1990². Même lorsqu'il reprend le texte de Juste la fin du monde dans Le Pays lointain, Lagarce simplifie son principe d'écriture.

5 De manière synthétique, on pourrait donc distinguer trois régimes principaux d'écriture chez Lagarce, en fonction des modalités des retours à la ligne au sein des répliques :

- un principe d'articulation essentiellement logique, emprunté à l'écriture en prose, jusqu'à Derniers remords avant l'oubli ;

- un régime d'écriture spécifique faisant système à partir de Music-hall, organisé sur les versets ;

- une valorisation expressive ponctuelle à partir de Nous les héros.

On pourrait encore affiner ce découpage, mais il paraît indubitable que Lagarce n'est jamais allé aussi loin dans l'exploration de ce régime spécifique d'écriture que dans Juste la fin du monde où la très grande majorité des vers tient sur une à deux lignes. Comment Lagarce en est-il venu à ce type d'écriture ? Quel sens faut-il lui donner? Les brouillons conservés peuvent nous aider à le comprendre.

\section{Naissance du verset lagarcien}

7 Depuis Les Prétendants écrits en 1988, Lagarce disposait d'une machine à écrire électronique permettant une présentation justifiée de son texte. Cette donnée matérielle a vraisemblablement joué un rôle dans les nouvelles dispositions de son texte sur l'espace de la page à partir de la pièce suivante, d'autant qu'il adopte une présentation singulière pour un texte de théatre : il isole toujours les noms des personnages, notés à gauche de la page, de leurs interventions qui occupent, plus à droite, les $3 / 4$ de la largeur en une sorte de colonne non matérialisée. Contrairement à la pratique claudélienne, cette disposition formelle ne s'impose pas de manière spontanée et généralisée dans le mouvement même de l'écriture: pour Music-hall, on dispose d'une première version en prose avant la version en versets. C'est différent pour Histoire d'amour (derniers chapitres), d'emblée écrite en versets, mais cette œuvre est une variation sur Histoire d'amour (repérages) qui était écrite en prose. 
8 Pour Juste la fin du monde, la perspective est plus complexe. Un brouillon de monologue non repris, attribué à «Lui » - et sans doute écrit au début de $1989^{3}$-, fait déjà usage de retours à la ligne réguliers en cours de phrase, alors même que la présentation du texte n'est pas justifiée. La première version encore inachevée de Juste la fin du monde dont on dispose, écrite lors du premier semestre de 1990 pendant son séjour à Berlin, est essentiellement rédigée en prose, selon le principe d'articulation logique en paragraphes déjà connu pour les répliques longues tendant au soliloque. Cependant, quelques passages adoptent la disposition en versets : c'est le cas de la première scène qui deviendra le Prologue de la version éditée. Son texte, plus court que dans la version définitive, est déjà formé d'une seule phrase qui s'organise en neuf versets de taille très variable allant d'un mot à neuf lignes. Le second monologue, à la scène 6 , poursuit cette exploration dans des versets n'excédant jamais six lignes. En revanche, on revient à une structuration davantage logique dans le troisième monologue de la scène 11 , alors que c'est le soliloque de la mère à la scène 8 qui, vers la fin, opte pour une disposition en versets :

Ce qu'ils veulent, ce qu'ils voudraient, c'est que tu les encourage[s], peut-être, que tu leur interdise[s] ou que tu les autorise[s], que tu dises à Suzanne de venir te rendre visite, ou qu'elle pourra, ou pourrait encore, qu'elle pourra, si l'envie lui vient, te rendre visite, qu'elle peut aller là où tu vis, maintenant - et nous ne savons pas vraiment où tu vis qu'elle peut bouger et partir et revenir encore [.... $]^{4}$

Par ailleurs, dans l'ensemble de cette première version, on note un soin particulier apporté à la présentation des interventions longues avec la constitution de paragraphes tantôt marqués par un simple retour à la ligne, tantôt par un saut de ligne. De plus, le texte dactylographié comporte des corrections manuscrites; on voit ponctuellement apparaitre des crochets ouverts («[») qui indiquent des retours à la ligne supplémentaires au moment de la relecture de son texte. Ces crochets interviennent d'abord entre des phrases - voire semblent contraindre Lagarce à changer la ponctuation pour commencer une nouvelle phrase ${ }^{5}$ - mais on en trouve à cinq reprises au sein de phrases dans la scène 8 de la première partie, signe du statut particulier ainsi accordé au soliloque de la mère.

\section{La seconde version inachevée de Juste la fin du monde}

10 Ce jeu ponctuel sur une disposition en versets qui valorise certains monologues ou soliloques se poursuit et s'intensifie dans la seconde version toujours inachevée, accompagnant à présent le mouvement d'écriture par amplification qui est le processus créateur régulier chez Lagarce. Le début du prologue le manifeste clairement : 


\begin{tabular}{|c|c|}
\hline $1^{\text {re }}$ version & $2^{\mathrm{e}}$ version \\
\hline $\begin{array}{l}\text { Après, l'année d'après - j'allais mourir à } \\
\text { mon tour - j'ai près de trente-quatre ans } \\
\text { maintenant et c'est à cet âge que je } \\
\text { mourrai, } \\
\text { I'année d'après, de nombreux mois déjà } \\
\text { que j'attendais à ne rien faire, à tricher et } \\
\text { ne plus savoir, que j'attendais d'en avoir } \\
\text { fini, } \\
\text { l'année d'après [...] }\end{array}$ & $\begin{array}{l}\text { Plus tard, l'année d'après } \\
\text { - j'allais mourir à mon tour - } \\
\text { j'ai près de trente-quatre ans maintenant et } \\
\text { c'est à cet âge que je mourrai, } \\
\text { l'année d'après, } \\
\text { de nombreux mois déjà que j'attendais à ne } \\
\text { rien faire, à tricher et ne plus savoir, } \\
\text { de nombreux mois que j'attendais d'en avoir } \\
\text { fini, } \\
\text { l'année d'après [...] }\end{array}$ \\
\hline
\end{tabular}

11 Cette scène commence à trouver sa rythmique spécifique avec les retours à la ligne qui marquent la logique syntaxique et sémantique, tout en donnant à voir les relances du discours et la perception des linéaments de la pensée des personnages avec différents plans contrapuntiques. On passe ainsi de 9 à 13 versets dans le prologue. Cependant, seul le début de cette scène est véritablement retravaillé dans cet esprit et, dans la suite du texte, ces retours à la ligne restent rares : le monologue suivant de Louis, ou les soliloques de Suzanne, Catherine, la Mère et Antoine en tirent occasionnellement parti. On dispose néanmoins de versions successives des p. 30 à 32 de la seconde version qui témoignent, au moment de l'« Intermède », du développement des effets de disposition, y compris pour des répliques assez courtes.

p. 31

\begin{tabular}{|l|l|}
\hline \multicolumn{1}{|c|}{$1^{\text {re }}$ version de la p. 31} & \multicolumn{1}{c|}{$2^{\mathrm{e}}$ version de la p. 31} \\
\hline Suzanne: & Suzanne : \\
Ce n'était pas si loin, et il aurait pu venir & Oui. \\
nous voir plus souvent, et rien de tragique & Ce n'était pas si loin, et il aurait pu venir \\
non plus, pas de drames ou de trahisons, & nous voir plus souvent, \\
cela que je ne comprends pas, ou ne peux & $\begin{array}{l}\text { et rien de tragique non plus, } \\
\text { pas comprendre. }\end{array}$ \\
& $\begin{array}{l}\text { cela que je ne comprends pas, ou ne peux } \\
\text { pas comprendre. }\end{array}$ \\
\hline
\end{tabular}

12 Pourtant, il serait trop simple de présenter le mouvement de l'écriture de Lagarce comme une généralisation de cette disposition: on peut également trouver des moments où il parait y renoncer :

p. 32

\begin{tabular}{|c|c|}
\hline $2^{\mathrm{e}}$ version de la p. 32 & $\begin{array}{c}\text { p. } 32 \text { dans la } 2^{\mathrm{e}} \text { version encore inachevée } \\
\text { de Juste la fin du monde }\end{array}$ \\
\hline $\begin{array}{l}\text { Catherine: } \\
\text { Les autres. } \\
\text { Je n'entends plus personne, } \\
\text { vous vous disputiez, je ne me trompe pas, } \\
\text { on entendait Antoine s'énerver, } \\
\text { c'est maintenant comme s'il n'y avait plus } \\
\text { personne et que nous nous soyons perdus } \\
\text { les uns les autres, } \\
\text { égarés, } \\
\text { je ne sais pas. }\end{array}$ & $\begin{array}{l}\text { Catherine: } \\
\text { Eux, les autres. Je n'entends plus personne, } \\
\text { vous vous disputiez, je ne me trompe pas, } \\
\text { on entendait Antoine s'énerver, et c'est } \\
\text { maintenant comme si tout le monde était } \\
\text { parti et que nous nous soyons perdus. }\end{array}$ \\
\hline
\end{tabular}


13 Il faudra attendre la version définitive de Juste la fin $d u$ monde - la $3^{\mathrm{e}}$ version reconnue dans l'ensemble des archives textuelles de cette œuvre - pour que l'intervention de Catherine - dont le texte ne change pratiquement pas - trouve sa disposition en versets.

\section{Une modalité d'écriture à part entière}

La deuxième version du texte de la pièce restait ainsi sur un principe de valorisation ponctuelle qui, dans la troisième version, devient principe global d'écriture: dans l'édition, le plus long paragraphe comporte sept lignes ${ }^{6}$, alors que ceux de quatre lignes sont déjà exceptionnels par rapport à ceux d'une à trois lignes qui dominent très largement. De plus, dans le contexte, ces cas limites de quatre lignes et plus, loin d'être assimilés à de la prose, apportent une forme de densité à un discours alors rassemblé dans un même élan énonciatif.

On doit à Michel Favriaud et Françoise Mignon une excellente étude sur « La ponctuation dans Juste la fin du monde $»^{7}$. Ils distinguent la «ponctuation noire » de la "ponctuation blanche », caractérisée par tous les effets de disposition du texte sur la page vierge, tout en soulignant la richesse constante de leur interaction. Lagarce ne se sert pratiquement jamais du verset - souvent court chez lui - comme Claudel qui aimait ponctuellement couper la phrase à des endroits inattendus afin de jouer sur des moments de tension du discours: il s'agissait de produire une "hémorragie du sens inclus" manifestant la «tension » inscrite dans le langage parlé. des ruptures syntaxiques plus ou moins marquées, la disposition n'a rien de neutre en ce qu'elle vient constituer des unités nouvelles que Michel Favriaud et Françoise Michon analysent selon différentes perspectives :

- une approche rythmique et prosodique ;

- une approche phono-syntaxico-sémantique ;

- une approche syntaxique ;

- une approche sémantique.

Cette ponctuation blanche participe ainsi d'une «sémantique plurielle» avec, comme élément sans doute fondamental, le fait que chaque unité formée corresponde à un " mouvement énonciatif $»^{9}$ du personnage qui met en jeu l'interprétation de son texte et, sur scène, la restitution du discours: "L'architecturation visuelle et phonique du discours, par la ponctuation blanche notamment, a une intensité et un pouvoir d'hétérogénéité énonciative et pragmatique potentiellement plus fort que n'a la segmentation linéaire actualisée par la ponctuation noire ${ }^{10}$.» Elle manifeste l'engendrement de la parole sur le principe régulier d'une résonance interne du discours, jouant tour à tour - voire simultanément - sur la continuité - notamment autour de la figure de l'épanorthose - et sur la discontinuité.

\section{Un renoncement?}

17 Pourtant, dès que Lagarce entreprend, à une date indéterminée, ce qu'il appelle d'abord une « seconde version » de Juste la fin du monde - qui deviendra Le Pays lointain -, il revient à un usage simplement ponctuel des effets de disposition, même dans le début du «Prologue », déjà inscrit dans une forme dialoguée : 


\begin{tabular}{|c|c|}
\hline Version définitive de Juste la fin du monde & $\begin{array}{l}\text { Tentative incomplète de réécriture de } \\
\text { Juste la fin du monde }\end{array}$ \\
\hline $\begin{array}{l}\text { Louis : } \\
\text { Plus tard, l'année d'après, } \\
\text { - j'allais mourir à mon tour - } \\
\text { j'ai près de trente-quatre ans maintenant et } \\
\text { c'est à cet âge que je mourrai, } \\
\text { l'année d'après, } \\
\text { de nombreux mois déjà que j'attendais à ne } \\
\text { rien faire, à tricher, à ne plus savoir, } \\
\text { de nombreux mois que j'attendais d'en avoir } \\
\text { fini, } \\
\text { l'année d'après, } \\
\text { comme on ose bouger parfois, } \\
\text { à peine, danger } \\
\text { devant un extrême, } \\
\text { imperceptiblement, sans vouloir faire de bruit } \\
\text { ou commettre un geste trop violent qui } \\
\text { réveillerait l'ennemi et vous détruirait } \\
\text { aussitôt, } \\
\text { l'année d'après, } \\
\text { malgré tout, } \\
\text { [...] } \\
\text { je décidai de retourner les voir, revenir sur } \\
\text { mes pas, aller sur mes traces et faire le } \\
\text { voyage [...] }\end{array}$ & $\begin{array}{l}\text { Louis : } \\
\text { Plus tard, l'année d'après, } \\
\text { Longue Date : Une année après que je } \\
\text { meurs, que je sois mort? } \\
\text { Louis: Exactement ça. } \\
\text { L'année d'après, } \\
\text { j'étais resté, là, seul, abandonné, toutes } \\
\text { ces sortes de choses, } \\
\text { plus tard, l'année d'après, } \\
\text { - j'allais mourir à mon tour - } \\
\text { (j'ai près de trente-quatre ans maintenant } \\
\text { et c'est à cet âge que je mourrai) } \\
\text { l'année d'après, je décidai de revenir ici. } \\
\text { Il y a encore ma famille qui vit dans ce } \\
\text { coin là, je vais aller les voir, je dis ça, } \\
\text { parler avec eux, régler cette affaire, ce } \\
\text { qu'on n'a pas dit et qu'on souhaite dire } \\
\text { avant de disparaître - on ne le gardera pas } \\
\text { dans la tête, on s'en débarrassera - je } \\
\text { ferai ce voyage et ensuite, j'en aurai } \\
\text { terminé, je rentrerai chez moi et } \\
\text { j'attendrai. Je serai paisible. }\end{array}$ \\
\hline
\end{tabular}

18 Par les italiques, comme par les incises signalées par des parenthèses ou des tirets déjà habituelles chez lui, il trouve un équivalent partiel, dans la ponctuation noire, à ce qu'apportaient ou confortaient les retours à la ligne dans la ponctuation blanche. En même temps, il renonce, dans ce passage réécrit, à la densité de la version de 1990, avec son unique phrase ou son imprécision frustrante et passionnante - l'année d'après quoi ? - pour dire plus explicitement les choses - l'histoire, la famille et les règlements de compte, l'homosexualité - perdant aussi sans doute au passage la force classique de la litote, de l'implicite et du non-dit, d'une poésie presque racinienne, visant à l'universel, de la précédente version.

On le sait, l'échec de Juste la fin du monde en 1990 auprès de ses destinataires a marqué très profondément le dramaturge qui renonce alors à l'écriture dramatique pour plusieurs années. Ce traumatisme se mesure jusqu'au choix du mode d'écriture : cette œuvre, dans la continuité de Music-hall et d'Histoire d'amour (derniers chapitres), offrait un nouveau visage à l'écriture lagarcienne, une rythmique donnant corps aux pulsions énonciatives de ses personnages qui, par la suite, a dû lui apparaître comme une préciosité poétique finalement anecdotique. Pourtant, elle contribue aujourd'hui certainement à la fascination contemporaine pour ce texte, aujourd'hui davantage lu, étudié et joué que Le Pays lointain, accomplissement testamentaire de l'œuvre lagarcienne. 


\section{NOTES}

1. Le texte en voix off de Catherine, constituant la seconde partie de La Photographie (1986), comporte déjà des retours à la ligne au milieu de phrases démesurées, mais il fait alors figure d'hapax. Sur sa dactylographie, Lagarce matérialise alors les retours à la ligne par des alinéas, ce qui apparaît également comme un hapax.

2. On lira avec attention les extraits reproduits du scénario Des Mensonges qui s'inscrit dans cette continuité malgré l'écriture à deux mains.

3. La machine utilisée semble la même, par la typographie, que celle qui a servi à taper la première version de Music-hall. Voir http://fanum.univ-fcomte.fr/lagarce/index.php?f=1\&d=128. On le sait, le projet de Juste la fin du monde - sous le titre Les Adieux - remonte au moins au début de 1988.

4. $1^{\text {re }}$ version, p. 14-15. Voir http://fanum.univ-fcomte.fr/lagarce/index.php?f=1\&d=12.

5. Il corrige « je ne crois pas avoir jamais connu leur père sans une voiture, avant même que nous nous marions, marrions?, avant qu'on ne soit mariés », en «je ne crois pas avoir jamais connu leur père sans une voiture. [Avant même que nous nous marions, marrions?, avant qu'on ne soit mariés » (Voir la première version p. 9, http://fanum.univ-fcomte.fr/lagarce/index.php? $\mathrm{f}=1 \& \mathrm{~d}=12)$.

6. Il se situe dans le soliloque de Suzanne, in Jean-Luc LAGARCE, Théâtre complet, tome III, Besançon, Les Solitaires intempestifs, 1999, p. 220.

7. Françoise MIGNON et Michel FAVRIAUD, «La ponctuation dans Juste la fin du monde : une mise en pièce du discours ", in Les Représentations de l'oral chez Lagarce: continuité, discontinuité, reprise, Paris, L'Harmattan, « Academia », 2012, p. 65-86.

8. Voir Paul Claudel, "Réflexions et propositions sur le vers français », E̋uvres en prose, Paris, Gallimard, «Bibliothèque de la Pléiade », 1965, p. 6. On sait que Claudel, dans Tête d'Or ou La Ville a pu aller exceptionnellement jusqu'à changer de verset au sein d'un mot.

Dans Juste la fin du monde, L'hapax serait ce passage de la scène 3 de la seconde partie, dans un soliloque d'Antoine, où il évoque son état malheureux par sympathie avec son frère : " comme toujours les plus jeunes frères se croient obligés de / l'être par imitation et inquiétude » (Juste la fin $d u$ monde, p. 97). On peut naturellement y voir une valorisation expressive au moment du renversement dramatique majeur de la pièce.

9. Françoise MIGNON et Michel FAVRIAUD, « La ponctuation dans Juste la fin du monde : une mise en pièce du discours ", art. cit., p. 80.

10. Ibid., p. 84.

\section{INDEX}

Mots-clés : Jean-Luc Lagarce, Juste la fin du monde, Le Pays lointain, Les Adieux, Music-Hall 


\section{AUTEURS}

\section{PASCAL LÉCROART}

Pascal Lécroart est professeur de Littérature française à l'Université de Franche-Comté où il dirige le Pôle « Arts et littérature » au sein d'ELLIADD (EA 4661). Spécialiste de Paul Claudel et de son rapport à la musique, il a publié différentes études sur les relations entre littérature, théâtre et musique au $\mathrm{Xx}^{\mathrm{e}}$ siècle. Il est responsable du Fonds numérique d'archives Jean-Luc Lagarce hébergé par FANUM. 